\title{
Orientation-Independent Differential Interference Contrast
}

\section{Microscopy}

\author{
Michael Shribak, and Shinya Inoué \\ Marine Biological Laboratory, Woods Hole, MA 02543, USA
}

\section{Abstract}

The image in a regular DIC microscope reflects the orientation of the prism shear direction and the optical path gradients in a phase specimen. If the shear direction lies parallel to the specimen boundary no contrast is generated. Also a bias retardance is generally introduced, which creates a gray background and reduces image contrast. Here we describe the theoretical foundation for a new DIC technique, which records phase gradients independently of their orientation and with the digitally generated gradient magnitude image as well as the optical path distribution image free from the gray background. Separate images can show the magnitude distribution of the optical path gradients and of the azimuths, or the two images can be combined into one picture e.g., with the brightness representing magnitudes and color showing azimuths respectively.

For experimental verification of the proposed technique we investigated various specimens such as glass rods embedded in Permount, Siemens star nano-fabricated in 90-nm thick silicon oxide layer, Bovine pulmonary artery endothelial cell, etc, using regular DIC optics on a microscope equipped with a precision rotating stage. Several images were recorded with the specimen oriented in different directions, but with the prism bias unchanged, followed by digital alignment and processing of the images. The results demonstrate that the proposed DIC technique can successfully image and measure phase gradients of transparent specimens, independent of the 
directions of the gradient. The orientation-independent DIC data obtained can also be used to compute the quantitative distribution of specimen phase or to generate enhanced, regular DIC images with any desired shear direction.

We are currently developing a new device using special DIC prisms, which allows the bias and shear directions to be switched rapidly without the need to mechanically rotate the specimen or the prism (US Patent Application 2005-0152030). With the new system an orientation independent DIC image should be obtained in a fraction of a second. A detailed description of the new system will be given in a future publication.

OCIS codes: $180.0180,100.2980,170.1530,170.3010,170.3890,170.3880$

\section{Introduction:}

Differential interference contrast light microscopy (DIC) is widely used to observe structure and motion in unstained living cells and isolated organelles. DIC microscopy uses an interference optical system in which the reference beam is sheared laterally by a small amount, generally by somewhat less than the diameter of the Airy disk. The technique produces a monochromatic shadow-cast image that displays the gradients of optical paths in the specimen. Those regions of the specimen where the optical paths increase along a reference direction appear brighter (or darker), while regions where the path differences decrease appear in reverse contrast. As the gradient of optical path grows steeper, image contrast is accordingly increased. Another important feature of DIC imaging is that it produces effective optical sectioning ${ }^{1}$. This is particularly obvious when high numerical aperture (NA) objectives are used together with high NA condenser illumination. 
The DIC technique was originally invented by F. H. Smith ${ }^{2}$ in 1947 . He placed between a pair of polarizers one Wollaston prism at the front focal plane of the condenser and another Wollaston prism in the back focal plane of objective lens. The first Wollaston prism splits the input beams angularly into two orthogonally polarized beams. The condenser makes the beam axes parallel with a small shear. Then the objective lens joins them in the back focal plane where the second Wollaston prism introduces an angular deviation into the beam and makes them parallel. Thereafter each of the two orthogonally polarized beams are recombined into one beam. This optical configuration creates a polarizing shearing interferometer, by which one visualizes phase gradients of the specimen under investigation.

In conventional medium- to high-NA objective lenses, the back focal plane is located inside the lens system and therefore not available for insertion of a Wollaston prism. If the Wollaston prism is placed far from the back focal plane, the prism only makes the rays parallel, but those beams are spatially displaced and hence are not recombined. Therefore the Smith DIC scheme requires special design for the microscope objective lenses so that the Wollaston prism can be incorporated within.

G. Nomarski, who proposed a modified Wollaston prism in 1952, provides an alternate solution $^{3,4}$. The Nomarski prism simultaneously introduces a spatial displacement and angular deviation of the orthogonally polarized beams. The prism therefore can be placed outside of the objective lens. By using crystal wedges with appropriately oriented axes, the Nomarski prism recombines the two beams (separated by the condenser Wollaston) as though a regular Wollaston prism were located in the proper plane in the objective lens. The Nomarski DIC scheme can be used in conjunction with regular high NA microscope objectives, which exhibit low stress birefringence. 
Video-enhanced DIC allows enchantment of contrast while removing unwanted background signal (such as fixed image noise due to dust particles, non-uniform illumination or other imperfections in the optical system) by subtraction of a reference image with no specimen ${ }^{5}$. Also S. Inoué and R.D. Allen et al., applied similar enhancement techniques to improve contrast in polarization microscopy $^{6,7}$.

G. Holzwarth et al. developed video-enhanced DIC microscopy with polarization modulation ${ }^{8,9}$. By switching the polarization of the incident light in alternate video frames with a computercontrolled liquid crystal variable retarder, the contrast signal of unstained biological specimens is increased by a factor of two, relative to "standard" video-enhanced DIC. The modulator switches image highlights into shadows and vice versa. By subtracting alternate frames, a difference DIC image is created in which contrast is doubled while image defects and noise are cancelled.

These regular DIC techniques show the two-dimensional distribution of optical path gradients as observed for a particular shear direction. The contrast of DIC images is thus not symmetrical, and varies proportionally with the cosine of the angle between by the azimuth of the specimen gradient and the direction of the wavefront shear. It is therefore prudent to examine unknown objects at several azimuths ${ }^{4,10}$.

C.Preza used 2, 4, or 8 conventional DIC images at different shear directions but with the same bias in an iterative phase-estimation method in order to calculate the specimen's phase function $^{11}$. She did not use images with the inverse biases because she supposed that they can be useful in reduction of noise only. The proposed theory was simulated by mathematical models employing large shear amount (ca. Airy radius) and large bias $\sim \pi / 2$. Thin specimen with $0.7 \mu \mathrm{m}$ thickness was investigated with an 0.5-NA objective lens. 
In order to overcome the limitations of existing systems we devised an orientation-independent DIC system, which requires no mechanical movement of the DIC prisms or of the specimen ${ }^{12}$. To explore the utility of such a system using immediately available components, we recorded several images with the specimen oriented in different directions, followed by digital alignment and processing of the images. Through computation we generate two separate images, which show the magnitude distribution of the optical path gradients and their azimuths. As shown in Section 3 the two images can be viewed separately or combined into one color shadow-cast picture e.g., with the brightness representing magnitude and color showing azimuth, respectively. The images obtained are independent of specimen orientation and free from the gray background. Furthermore, we show how it is possible to generate quantitative phase images (showing distribution of optical paths) as well as enhanced regular DIC images with any shear direction from the orientation-independent DIC data.

\section{Principles of orientation-independent DIC microscopy}

\subsection{Theoretical model of DIC image}

Schematic of ray paths in a DIC microscope is shown in Fig.1. The polarizer creates a linearly polarized beam, which contains two components $\mathrm{E}_{\mathrm{x}}$ and $\mathrm{E}_{\mathrm{y}}$ with equal intensities and the retarder introduces a variable phase shift $\Gamma$ between the components. Then the first Wollaston prism splits the beam and the second Wollaston prism combines the split beam back as described in the previous section.

A DIC image can be modeled as the superposition of one image over an identical copy that is displaced by a differential amount $\delta$ and phase shifted by bias $\Gamma$. For example, a point $\mathrm{A}$ in the first image will be superimposed with point B in the copy of the image (See Fig. 1). For 
simplicity consider a pure (non-birefringent) phase specimen, which is described in a Cartesian coordinates $\mathrm{XOY}$ in the object plane. If the optical path distribution in the first image is $\phi(x, y)$, then the second displaced image has the optical path distribution: $\phi\left(x-\delta_{x}, y-\delta_{y}\right)$. Here $\delta_{x}$ and $\delta_{y}$ are projections of shear vectors on the corresponding coordinate axes. The optical paths difference $(\boldsymbol{O P D})$ between the two images can then be written as:

$$
O P D(x, y)=\phi(x, y)-\phi\left(x-\delta_{x}, y-\delta_{y}\right)
$$

Taking into account that the shear distance is a small value (ca. half of the optical resolution limit), the optical path distribution in the displaced copy $\phi\left(x-\delta_{x}, y-\delta_{y}\right)$ can be written in the following linear approximation:

$$
\phi\left(x-\delta_{x}, y-\delta_{y}\right)=\phi(x, y)-(\vec{\nabla} \phi(x, y), \vec{\delta})
$$

where the last term on the right-hand side is the scalar product of two vectors:

$\vec{\nabla} \phi(x, y) \equiv \operatorname{grad}(\phi(x, y))=\left(\frac{\partial \phi(x, y)}{x}, \frac{\partial \phi(x, y)}{y}\right)$ is a gradient of optical path, and $\vec{\delta}=\left(\delta_{x}, \delta_{y}\right)$ is a shear vector.

Thus a phase difference distribution between two overlapping images $\varphi(x, y)$ is described by the formula:

$$
\varphi(x, y)=\frac{2 \pi}{\lambda}(\Gamma+(\vec{\nabla} \phi(x, y), \vec{\delta}))=\frac{2 \pi}{\lambda}(\Gamma+\delta \chi(x, y) \cos \psi(x, y))
$$

where $\lambda$ is wavelength, $\psi(x, y)$ is angle between the shear direction and the optical path gradient, and $\gamma(x, y)=|\vec{\nabla} \phi(x, y)|$ is the gradient magnitude.

The intensity distribution in the image, which is the result of interference of the two beams, is: 


$$
I(x, y)=\frac{1}{2} \tilde{I}\left(1-\cos \left(\frac{2 \pi}{\lambda}(\Gamma+\delta \chi(x, y) \cos (\theta(x, y)-\sigma))\right)\right)+I_{\min }
$$

where $\tilde{I}$ is the beam intensity before entering the first DIC prism, $\theta(x, y)$ is the gradient azimuth, and $\sigma$ is the shear azimuth (here we used $\psi(x, y)=\theta(x, y)-\sigma)$.

From formula (4), if the shear direction coincides with the optical path gradient $\left(\psi=0^{\circ}\right.$ or $\psi=180^{\circ}$ ) the picture contrast is maximal. In case shear direction is oriented perpendicular to the gradients $\left(\psi=90^{\circ}\right.$ or $\left.\psi=270^{\circ}\right)$ the intensity contrast becomes zero.

The equation does not take into consideration the refraction of beam in a thick non-homogeneous specimen or change of intensities and phase due to birefringence. Therefore quantitative interpretation of the results will work better with a thin low-birefringent specimen.

\subsection{Six-frame processing algorithm}

In order to find the two-dimensional distribution of magnitude and azimuth of optical path gradients we can apply a technique similar to the one, which we developed for orientation independent polarization microscopy ${ }^{13}$. For instance, we take the next four raw images with $90^{\circ}$ step differences in the shear orientation and with the same bias $\Gamma$, and two raw images with no bias:

$$
\begin{array}{ll}
\sigma=0^{\circ}: & I_{1}=\frac{1}{2} \tilde{I}\left(1-\cos \left(\frac{2 \pi}{\lambda}(\Gamma+\delta \gamma \cos \theta)\right)\right)+I_{\min }, \\
\sigma=90^{\circ}: & I_{2}=\frac{1}{2} \tilde{I}\left(1-\cos \left(\frac{2 \pi}{\lambda}(\Gamma+\delta \gamma \sin \theta)\right)\right)+I_{\min } \\
\sigma=180^{\circ}: & I_{3}=\frac{1}{2} \tilde{I}\left(1-\cos \left(\frac{2 \pi}{\lambda}(\Gamma-\delta \gamma \cos \theta)\right)\right)+I_{\min },
\end{array}
$$




$$
\begin{array}{ll}
\sigma=270^{\circ}: & I_{4}=\frac{1}{2} \tilde{I}\left(1-\cos \left(\frac{2 \pi}{\lambda}(\Gamma-\delta \gamma \sin \theta)\right)\right)+I_{\min }, \\
\sigma=0^{\circ} \text { or } \sigma=180^{\circ}: & I_{0}{ }^{\prime}=\frac{1}{2} \tilde{I}\left(1-\cos \left(\frac{2 \pi}{\lambda} \delta \gamma \cos \theta\right)\right)+I_{\min }, \\
\sigma=90^{\circ} \text { or } \sigma=270^{\circ}: & I_{0}{ }^{\prime \prime}=\frac{1}{2} \tilde{I}\left(1-\cos \left(\frac{2 \pi}{\lambda} \delta \gamma \sin \theta\right)\right)+I_{\min } .
\end{array}
$$

Then compute the following two terms:

$$
\begin{gathered}
A \equiv \frac{I_{1}-I_{3}}{I_{1}+I_{3}-2 I_{0}{ }^{\prime}} \tan \left(\frac{\pi \Gamma}{\lambda}\right)=\tan \left(\frac{2 \pi}{\lambda} \delta \gamma \cos \theta\right), \\
B \equiv \frac{I_{2}-I_{4}}{I_{2}+I_{4}-2 I_{0}{ }^{\prime \prime}} \tan \left(\frac{\pi \Gamma}{\lambda}\right)=\tan \left(\frac{2 \pi}{\lambda} \delta \gamma \sin \theta\right) .
\end{gathered}
$$

Using these terms we can find the exact solution for gradient magnitude and azimuth distributions $\gamma(x, y)$ and $\theta(x, y)$ :

$$
\begin{aligned}
& \chi(x, y)=\frac{\lambda}{2 \pi \cdot \delta} \sqrt{(\arctan A)^{2}+(\arctan B)^{2}}, \\
& \theta(x, y)=\arctan \left(\frac{\arctan B}{\arctan A}\right) .
\end{aligned}
$$

\subsection{Four-frame processing algorithm}

In some cases, fewer than six images are collected to support calculation of orientationindependent values and images. Some approximations may be required to complete the calculations. For example, if the product of shear distance and gradient magnitude is small $\left(\frac{2 \pi}{\lambda} \delta \gamma<<1\right)$ formula (4) can be simplified to: 


$$
I(x, y)=\frac{1}{2} \tilde{I}\left(1-\cos \left(\frac{2 \pi}{\lambda} \Gamma\right)+\frac{2 \pi}{\lambda} \delta \gamma \sin \left(\frac{2 \pi}{\lambda} \Gamma\right) \cos (\theta(x, y)-\sigma)\right)+I_{\min } .
$$

Then four images can be collected, and modeled as:

for $\sigma=0^{\circ}, \quad I_{1}=\frac{1}{2} \tilde{I}\left(1-\cos \left(\frac{2 \pi}{\lambda} \Gamma\right)+\frac{2 \pi}{\lambda} \delta \gamma \sin \left(\frac{2 \pi}{\lambda} \Gamma\right) \cos \theta\right)+I_{\min }$;

for $\sigma=90^{\circ}, \quad I_{2}=\frac{1}{2} \tilde{I}\left(1-\cos \left(\frac{2 \pi}{\lambda} \Gamma\right)+\frac{2 \pi}{\lambda} \delta \gamma \sin \left(\frac{2 \pi}{\lambda} \Gamma\right) \sin \theta\right)+I_{\min }$;

for $\sigma=180^{\circ}, \quad I_{3}=\frac{1}{2} \tilde{I}\left(1-\cos \left(\frac{2 \pi}{\lambda} \Gamma\right)-\frac{2 \pi}{\lambda} \delta \gamma \sin \left(\frac{2 \pi}{\lambda} \Gamma\right) \cos \theta\right)+I_{\min }$;

for $\sigma=270^{\circ}, \quad I_{4}=\frac{1}{2} \tilde{I}\left(1-\cos \left(\frac{2 \pi}{\lambda} \Gamma\right)-\frac{2 \pi}{\lambda} \delta \gamma \sin \left(\frac{2 \pi}{\lambda} \Gamma\right) \sin \theta\right)+I_{\min }$.

Thus the next two equations allow calculation of the two-dimensional distribution of the gradient magnitude and azimuth of optical paths in the specimen. These can be imaged separately and independently of the shear direction:

$$
\begin{gathered}
\chi(x, y)=\frac{\lambda}{2 \pi \cdot \delta} \tan \left(\frac{\pi \Gamma}{\lambda}\right) \sqrt{\left(\frac{I_{1}-I_{3}}{I_{1}+I_{3}}\right)^{2}+\left(\frac{I_{2}-I_{4}}{I_{2}+I_{4}}\right)^{2}}, \\
\theta(x, y)=\arctan \left(\frac{I_{2}-I_{4}}{I_{1}-I_{3}}\right) .
\end{gathered}
$$

Here we assumed that the microscope has moderately high extinction $\left(\tilde{I} / I_{\min }>200\right)$, and thus neglected $I_{\min }$ in the divisors of the first equation.

Notice that the two algorithms considered above employ ratios between intensities of light that has interacted with the specimen. Therefore they suppress contributions of absorption by the specimen, nonuniformity of illumination, etc., which can otherwise deteriorate a DIC image. 


\subsection{Two-frame processing algorithm}

Other numbers of specimen images, for instance 2 or 3, can be also used for calculating magnitude and azimuth of the gradient. For example, two specimen images and three background images can be collected, and modeled accordingly to formula (8):

for $\sigma=0^{\circ}, \quad I_{1}=\tilde{I} \sin \left(\frac{\pi}{\lambda} \Gamma\right)\left(\sin \left(\frac{\pi}{\lambda} \Gamma\right)+\frac{2 \pi}{\lambda} \delta \gamma \cos \left(\frac{\pi}{\lambda} \Gamma\right) \cos \theta\right)+I_{\min }$

for $\sigma=90^{\circ}, \quad I_{2}=\tilde{I} \sin \left(\frac{\pi}{\lambda} \Gamma\right)\left(\sin \left(\frac{\pi}{\lambda} \Gamma\right)+\frac{2 \pi}{\lambda} \delta \gamma \cos \left(\frac{\pi}{\lambda} \Gamma\right) \sin \theta\right)+I_{\min }$;

and background images without the specimen:

for $\sigma=0^{\circ}, \quad I_{b g 1}=\tilde{I} \sin ^{2}\left(\frac{\pi}{\lambda} \Gamma\right)+I_{\min }$

for $\sigma=90^{\circ}, \quad I_{b g 2}=\tilde{I} \sin ^{2}\left(\frac{\pi}{\lambda} \Gamma\right)+I_{\min }$

for $\Gamma=0$ and an arbitrary $\sigma, \quad I_{b g 0}=I_{\min }$.

Values A and B, are calculated as follows:

$$
A \equiv \frac{I_{1}-I_{b g 1}}{I_{b g 1}-I_{b g 0}} \tan \left(\frac{\pi}{\lambda} \Gamma\right)=\frac{\tilde{I} \frac{2 \pi}{\lambda} \delta \gamma \sin \left(\frac{\pi}{\lambda} \Gamma\right) \cos \left(\frac{\pi}{\lambda} \Gamma\right) \cos \theta}{\tilde{I} \sin ^{2}\left(\frac{\pi}{\lambda} \Gamma\right)} \tan \left(\frac{\pi}{\lambda} \Gamma\right)=\frac{2 \pi}{\lambda} \delta \gamma \cos \theta
$$

and

$$
B \equiv \frac{I_{2}-I_{b g 2}}{I_{b g 2}-I_{b g 0}} \tan \left(\frac{\pi}{\lambda} \Gamma\right)=\frac{\tilde{I} \frac{2 \pi}{\lambda} \delta \gamma \sin \left(\frac{\pi}{\lambda} \Gamma\right) \cos \left(\frac{\pi}{\lambda} \Gamma\right) \sin \theta}{\tilde{I} \sin ^{2}\left(\frac{\pi}{\lambda} \Gamma\right)} \tan \left(\frac{\pi}{\lambda} \Gamma\right)=\frac{2 \pi}{\lambda} \delta \gamma \sin \theta
$$


Then the gradient magnitudes $\chi(x, y)$ and azimuth distributions $\theta(x, y)$ can be obtained using the next formulae:

$$
\begin{gathered}
\chi(x, y)=\frac{\lambda}{2 \pi \delta} \sqrt{A^{2}+B^{2}}, \\
\theta(x, y)=\arctan \left(\frac{B}{A}\right) .
\end{gathered}
$$

Further approximations can be employed, for example, to simplify the calculations or to reduce the number of collected background images. For example, if $I_{b g 0}$ is small it can be omitted in the above equations.

Using just two specimen images for obtaining orientation-independent DIC data increases the speed of measurement. However the sensitivity becomes lower. Moreover the algorithm doesn't use ratios between intensities of light, which has interacted with the specimen. Therefore this particular algorithm can be used only when the specimen absorption is negligible, and illumination is very stable and uniform.

\subsection{Using orientation-independent DIC data to obtain enhanced regular DIC and phase images}

After computing the optical path gradient distribution we can restore enhanced regular DIC images $I_{e n h}(x, y)$ with any selected shear direction $\sigma$ :

$$
I_{e n h}(x, y)=1-\cos \left(\frac{2 \pi}{\lambda}(\Gamma+\delta \gamma(x, y) \cos (\theta(x, y)-\sigma))\right) .
$$

The enhanced image, $I_{e n h}(x, y)$, provides a calculated image for any desired shear direction $\sigma$ without the requirement to directly collect an image for that shear direction. Moreover the 
enhanced image will have less noise than a regular DIC image. In additional it suppresses deterioration of the image due to specimen absorption and illumination nonuniformity.

The distribution of optical phase $\Phi(x, y)$ in the specimen can be obtained by computing the following line integral ${ }^{14}$ :

$$
\Phi(x, y)=\frac{2 \pi}{\lambda} \int_{C}\left(\chi\left(x^{\prime}, y^{\prime}\right) \cos \theta\left(x^{\prime}, y^{\prime}\right) d x^{\prime}+\chi\left(x^{\prime}, y^{\prime}\right) \sin \theta\left(x^{\prime}, y^{\prime}\right) d y^{\prime}\right) .
$$

As the simplest contour for integration we can choose rectangular ones along the coordinate axes:

$$
\Phi(x, y)=\frac{2 \pi}{\lambda}\left(\int_{0}^{x} \gamma\left(x^{\prime}, 0\right) \cos \theta\left(x^{\prime}, 0\right) d x^{\prime}+\int_{0}^{y} \gamma\left(x, y^{\prime}\right) \sin \theta\left(x, y^{\prime}\right) d y^{\prime}\right) .
$$

Here we assign zero phase to the beginning point of the coordinate.

Where calculations are performed in terms of pixels, this expression can have the following form:

$$
\Phi_{m n}=\frac{2 \pi}{\lambda}\left(\sum_{k=1}^{n} \gamma_{0 k} \cos \theta_{0 k}+\sum_{p=1}^{m} \gamma_{p n} \sin \theta_{p n}\right)
$$

where $m$ and $n$ are row and column numbers of the pixels, and $\Phi_{m n}$ is the value of the optical phase for the pixel $m, n$.

Also other techniques for phase computation can be used, for instance, iterative computation ${ }^{11}$, non-iterative Fourier phase integration ${ }^{15}$, or nonlinear optimization with hierarchical representation $^{16}$, etc.

D.Biggs et al. $^{17}$ have developed iterative deconvolution approach for computation of phase image, on the same principles as deconvolution techniques normally used to remove out-of-focus 
haze $^{18}$. In the case of DIC imagery, if the image background is removed such that the data has a zero-mean, then the point spread function (PSF) can be modeled as a positive and negative Dirac delta function with small separation between them ${ }^{11}$. If the specimen is not a purely phase object and contains absorbing structures then four DIC images with 90-degree differences in orientation should be collected. The two sets of opposite orientations can be used to separate the phase difference information from the amplitude modulation, which reduces potential artifacts in the reconstruction. This pre-processing is also valuable when using small bias retardations to maximize image contrast, which also result in a very non-linear intensity response to phase differences.

The Biggs' optimization is based on minimizing the least squares error between the observed phase differences, and the recalculated DIC image from the estimated phase map. For a two image reconstruction, the errors from each are combined to form a single metric. The single phase image is iteratively solved by minimizing the error metric using a gradient decent optimization with acceleration ${ }^{19}$ with typically 100 iterations being required. A conjugate gradient, or other optimization approach could also be employed. Depending upon the specimen, a penalty term can also be introduced to minimize negative phase values with respect to the background, if that information is known a priori. The reconstruction process thus far did not take into account any effect of diffraction or other optical effects that limit the observable resolution in the image. Once the phase map is calculated, the image is then further deconvolved using an iterative blind deconvolution algorithm that is able to determine the amount of blur directly from the provided image, and use this PSF to improve the resolution of the image. Once the PSF is known, only 5 iterations are typically required. The algorithm is commercially available in the AutoDeblur software from AutoQuant Imaging (Troy, NY). 


\section{Experimental verification}

\subsection{Description of experiment}

For verification of theoretical analysis, we carried out several experiments using artificial and biological specimens. We employed the Universal (Polarized) Light Microscope (ULM or "ShinyaScope") that has been developed by one of us over 5 decades, and which is one of the most sophisticated and versatile light microscope platforms available today ${ }^{1}$. The microscope is optimized for polarization imaging, and includes a precision stage that allows rotation of the specimen with fractional microns of excentricity or focal shift. The ULM is equipped with a high brightness fiber illuminator. A 100 Watt concentrated mercury arc lamp coupled with green 546 $\mathrm{nm}$ bandpath filter was employed during the experiments. For the test published here, we used a Nikon 20x objective with NA 0.75 and a Nikon Universal Achromatic-Aplanat condenser with NA 1.4 stopped down to match the objective NA. Images were collected with a Hamamatsu C5985 chilled CCD camera or a Hamamatsu C4742-95 high resolution digital CCD camera ${ }^{20}$.

The resolution of the microscope, which is determined by the ratio $1.22 \lambda /(2 N A)$, was $0.44 \mu \mathrm{m}$, and depth of field according to the ratio $\lambda n / N A^{2}$ was $1.5 \mu \mathrm{m}$, where $\mathrm{n}$ is the refractive index of the objective lens immersion medium ${ }^{1}$.

We acquired four regular DIC images of the specimen with the same bias at $90^{\circ}$ orientation difference, and two images without the bias as described in the previous section. In addition, we took corresponding background DIC images without the specimen in the field of view. The regular DIC images were aligned using ImageJ software ${ }^{21}$. The background images were used to compensate nonuniformity in intensity distribution of the illumination beam, since otherwise 
each part of the specimen can be exposed to different light intensities during rotation of the specimen.

Using formulae (7), (15), and (18), we computed images of gradient magnitude and azimuth distributions of optical paths, optical phase distribution, and enhanced regular DIC image with any chosen shear direction. For the computation we used Mathematica software ${ }^{22}$.

\section{2. Orientation-independent DIC images of microscopic glass rods embedded in immersion medium}

At first we explored a model specimen, which is optically similar to transparent filaments in living organisms. We took short segments of glass rods, which are used as spacers in liquid crystal cells, and embedded them in Fisher Permount* Mounting Medium (Fisher Scientific Company L.L.C, Hampton, USA, http://www.fishersci.com). The refractive indices of the glass rods and Permount at wavelength $546 \mathrm{~nm}$ were measured with a Jamin-Lebedeff microscope (Zeiss, Germany). They measured 1.554 and 1.524 respectively. A drop of the suspension was placed between microscope slide and a $0.17 \mathrm{~mm}$ thick coverslip, and the preparation was placed on the precision rotating microscope stage.

One of the images taken by regular DIC optics is shown in Fig.2. Also the figure contains line scans of intensities in two sections, one of them (AA') is scanned along the shear direction and another (BB') is perpendicular the shear direction. Both scans are normalized to the maximal intensity in the image.

Grayscale images of gradient magnitude computed from equation (7) are given in Fig.3. Here brightness is linearly proportional to the magnitude. The absolute magnitude value wasn't determined because the amount of the exact shear was unknown. The gradient magnitude values 
measured along lines A-A' and B-B' are shown by dots (Fig.3, right). In contrast to Figure 2, the two linear scans of magnitude now exhibit virtually no difference. The gradient magnitude image clearly shows the rod boundaries independently of orientation.

For preliminary analysis of the experimental data we employed a simple model for DIC image of glass microspheres, as described by A.Resnick ${ }^{23}$. He used the model for analysis of DIC images of $2-\mu \mathrm{m}$ diameter microspheres with a 100x/1.4NA objective lens. The spherical model can be also used for a (one-dimensional) glass rod, which is oriented parallel to the object plane. This model does not take into account refraction of rays, microscope resolution, out-of-focus contributions in the thick specimen, change in polarization, etc.

Assume that the center of the Cartesian coordinate corresponds to the center of the rod, the Yaxis is parallel to the rod axis, and the $\mathrm{Z}$-axis is directed along the microscope axis. The relative phase retardation $\Phi$ (in radians) experiences by a ray traveling trough the rod along the microscope axis is as follows ${ }^{23}$ :

$$
\Phi(x)=\frac{4 \pi}{\lambda}\left(n_{r}-n_{m}\right) \sqrt{r^{2}-x^{2}}=\Phi_{\max } \sqrt{1-\left(\frac{x}{r}\right)^{2}},
$$

where $\Phi_{\max }=\frac{4 \pi}{\lambda}\left(n_{r}-n_{m}\right) r$,

$r$ is the diameter of the rod, and $n_{r}$ and $n_{m}$ are refractive indices of the rod and the surrounding media respectively.

When two rays are spatially displaced along the X-axis by a small distance $\delta<<r$, the gradient magnitude between them can be determined by the following formula: 


$$
x(x)=\frac{\Phi(x+\delta)-\Phi(x)}{\delta}=\frac{2}{\delta}\left(n_{r}-n_{m}\right) \times\left\{\begin{array}{l}
0, \text { if } x<-r \text { or } x \geq r+\delta \\
\sqrt{r^{2}-x^{2}}, \text { if }-r \leq x<-r+\delta \\
\left|\sqrt{r^{2}-x^{2}}-\sqrt{r^{2}-(x-\delta)^{2}}\right|, \text { if }-r+\delta \leq x<r \\
\sqrt{r^{2}-(x-\delta)^{2}}, \text { if } r \leq x<r+\delta
\end{array} .\right.
$$

The maxima of function $\gamma(x)$ occur at $x=-r+\delta$ and $x=r$. For $\delta<<r$ :

$$
\gamma_{\max }=2 \sqrt{2}\left(n_{r}-n_{m}\right) \sqrt{\frac{r}{\delta}}
$$

Then the normalized gradient magnitude can be written as:

$$
x(x) / \gamma_{\max }=\frac{1}{\sqrt{2 r \delta}} \times\left\{\begin{array}{l}
0, \text { if } x<-r \text { or } x \geq r+\delta \\
\sqrt{r^{2}-x^{2}}, \text { if }-r \leq x<-r+\delta \\
\left|\sqrt{r^{2}-x^{2}}-\sqrt{r^{2}-(x-\delta)^{2}}\right|, \text { if }-r+\delta \leq x<r \\
\sqrt{r^{2}-(x-\delta)^{2}}, \text { if } r \leq x<r+\delta
\end{array}\right.
$$

Theoretical plots of the normalized gradient magnitude are shown by the solid lines in Fig.3 (right). We used $r=2.1 \mu \mathrm{m}$ for the section A-A', and $r=2.0 \mu \mathrm{m}$ for the section B-B'. The shear distance introduced by the microscope DIC prisms was unknown and it was estimated to be 0.2 $\mu \mathrm{m}$ for these examples. The experimental results show a better correspondence for higher values of the gradient magnitude. However for lower values the experimental data are wider than the theoretical curve by ca. $0.5 \mu \mathrm{m}$. This fact indicates that the theoretical model needs to be improved taking into account the Airy disc radius $(0.44 \mu \mathrm{m}$ at $\mathrm{NA}=0.75)$, and the distance $(0.31$ $\mu \mathrm{m})$, between the beam and the contact points of the peripheral rays with the rod etc. 
Both gradient magnitude and azimuth images can be combined into one, color shaded picture e.g., with the brightness representing magnitude and color showing azimuth respectively (Fig.4). As can be seen from the shaded color, a gradient vector on rod boundaries is always oriented toward the rods. That means the rod's refractive index is higher than the refractive index of Permount.

Using the simple numerical contour integration described in formula (17), we computed the twodimensional phase distributions $\Phi(x, y)$ of rays transmitted through the glass rods (Figure 5 (left)). The reconstructed phase image contains some streaks, which can be caused by misalignment of the raw DIC images. The normalized phase values $\left(\Phi(x, y) / \Phi_{\max }\right)$ along lines A-A' and B-B' are shown by dots (Fig.5, right).

\section{3. Orientation-independent DIC imaged microstructure of MBL-NNF test} target

We investigated another man-made specimen: a test target developed us at the Marine Biological Laboratory, Woods Hole, Massachusetts in collaboration with the National Nanofabrication Facility at Cornell University ${ }^{24}$. The specimen has a 90-nm-thick $\mathrm{SiO}_{2}$ film deposited on a \#1 $1 / 2$ glass coverslip. The film was treated to remove the $\mathrm{SiO}_{2}$ from regions exposed to the electron beam by microlithography. Then the coverslip was cemented with the target directly lying against the inside surface of a $1 \mathrm{~mm}$ thick regular microscope slide. The target includes a $75 \mu \mathrm{m}$ diameter Siemens test star that consists of 36 wedge pairs. The period near the outer edge is 6.5 $\mu \mathrm{m}$, decreasing linearly toward the center. The optical path difference between the etched structure and the film was measured with a Jamine-Lebedeff microscope. It equals $60 \mathrm{~nm}$.

In Fig.6 we show a regular DIC images (left), computed grey scale gradient magnitude image 
(center), and phase (right) of the MBL-NNF test target. In the third panel, the dark star image and the light background points out that the refractive index in the star wedges is lower than in the surrounding silicon dioxide film. The lower portions of the figure show line scans of intensity in the regular DIC image, the gradient magnitude image and phase image along the same section. The plots are normalized to the maximum intensity values.

\section{4. Orientation-independent DIC images of fixed Bovine pulmonary artery endothelial cell}

The analytical technique has been used with several biological specimens. One of them was a prepared microscope slide (F-14780) containing bovine pulmonary artery endothelial cells stained with a combination of fluorescent dyes (Molecular Probes, Inc., Eugene, OR, USA http://www.probes.com).

Fig. 6 demonstrates a regular DIC image of a cell (left), computed gradient magnitude image (center) and computed phase image (right). The orientation independent DIC images clearly reveal the refractive boundaries and detailed structures of the cell. The phase image shows the dry mass distribution of the cell regions under investigation.

In Fig.7 we present enhanced regular DIC images of the same bovine epithelial cell with various shear directions at every $45^{\circ}$, which are computed from the gradient data. The images show the changes in contrast and grey shading of different parts of the cell as the computed shear direction is altered. In these digitally enhanced DIC images, the empty areas have $31 \%$ less optical noise than the original DIC images.

These results demonstrate that the proposed DIC technique can successfully image and measure phase gradients as well as distribution of phase retardances (optical path differences) of 
transparent specimens, independent of the directions of the gradient, and minimally influenced by specimen absorption.

\section{Conclusions:}

1. We have developed the theoretical basis for orientation-independent differential interference contrast (OI-DIC) microscopy. The new approach allows precise analyses of organelle morphology, motility, shape changes, as well dry mass distribution, e.g. in unstained living cells.

2. Using regular DIC optics and a microscope equipped with a precision rotating stage, we confirmed the theoretical validity of the proposed technique.

3. Since this article was submitted for publication, US Patent Application 2005-0152030 "Orientation-independent differential interference contrast microscopy technique and device" was published on July 14, 2005 ${ }^{12}$. The patent describes designs for achieving orientation independent DIC images using novel arrangements of optical and electro-optical components, without the need for any mechanical re-orientation or adjustments of the DIC prisms or of the specimen. Using the new device and the algorithms described in the current paper, we estimate that orientation-independent DIC images can be digitally generated in a fraction of a second.

\section{Acknowledgements:}

The authors thank Drs. David Biggs and Mike Meichle of AutoQuant Imaging Inc. (Troy, NY) for helpful discussions and for providing deconvoluted phase images. We are also grateful to Dr. Rudolf Oldenbourg of the Marine Biological Laboratory and Dr. James LaFountain of the University of Buffalo for their encouragement and support. 


\section{References:}

1. S. Inoué, and K.R. Spring, Videomicroscopy: The Fundamentals. 2nd edition (Plenum Press, New York, NY. 1997).

2. F. H. Smith, "Interference microscope", US Patent 2601175, patented June 17, 1952.

3. G. Nomarski, "Interferential polarizing device for study of phase object", US Patent 2924142, patented February 9, 1960.

4. R.D Allen, G.B. David, and G. Nomarski. "The Zeiss-Nomarski differential equipment for transmitted light microscopy", Zeitschrift für Wissenschaftliche Mikroscopie und Mickroskopische Technik, 69 (4), 193-221 (1969).

5. R.D. Allen, N.S. Allen, and J.L. Travis, "Video-enhanced contrast differential interference contrast (AVEC-DIC) microscopy: a new method capable of analyzing microtubule-related motility in the reticulopodial network of Allogromia laticollaris", Cell Motility, 1 (3), 291$302(1981)$

6. S. Inoué, "Video image processing greatly enhances contrast, quality, and speed in polarization-based microscopy", J. of Cell Biology, 89, 246-356 (1981).

7. R.D.Allen, J.L. Travis, N.S. Allen, and H.Yilmaz, "Video-enhanced contrast polarization (AVEC-POL) microscopy: a new method applied to detection of birefringence in motile reticulopodial network of Allogromia laticollaris", Cell Motility, 1 (3), 275-289 (1981).

8. G. Holzwarth, S.C. Webb, D.J. Kubinski, and N.S. Allen, "Improving DIC microcopy with polarization modulation”, J. Microscopy, 188 (Pt 3), 249-254 (1997).

9. G. Holzwarth, D.B. Hill, and E.B. McLaughlin, "Polarization-modulated differentialinterference contrast microscopy with a variable retarder", Applied Optics, 39, 6288-6294 (2000). 
10. M. Pluta, Advanced light microscopy. Vol.2: Specialized methods (Elsevier Science Publishing Co., Inc., New York, NY. 1989).

11. C. Preza, "Rotational-diversity phase estimation from differential-interference-contrast microscopy images", J. Opt. Soc. Am. A, 17, 415-424 (2000).

12. M.Shribak "Orientation-independent differential interference contrast microscopy technique and device”, US Patent Application 2005-0152030 (2005).

13. M.Shribak, and R. Oldenbourg, "Technique for fast and sensitive measurements of twodimensional birefringence distribution”, Applied Optics, 42, 3009-3017 (2003).

14. D.C.Ghiglia, M.D.Pritt, Two-dimensional phase unwrapping (John Wiley \& Sons, New York. 1998).

15. M.R. Arnison, K.G. Larkin, C.J.R. Sheppard, N.I. Smith, and C.J. Cogswell, "Linear phase imaging using differential interference contrast microscopy”, J. Microscopy, 214 (Pt 1), 7-12 (2004).

16. F.Kagalwala, T.Kanade, "Reconstructing specimens using DIC microscope images", IEEE Transactions on Systems. Man and Cybernetics - Part B: Cybernetics, 33, 728-737 (2003).

17. D.Biggs, private communication.

18. T. J. Holmes, S. Bhattacharyya, J. A. Cooper, D. Hanzel, V. Krishnamurthi, W. Lin, B. Roysam, D. H. Szarowski, and J. N. Turner, "Light microscopic images reconstructed by maximum likelihood deconvolution”. In: Handbook of Biological Confocal Microscopy, James B. Pawley, ed., (Plenum Press, New York, 1995), pp. 389-402.

19. D.S.C. Biggs, and M. Andrews, "Acceleration of iterative image restoration algorithms", Applied Optics, 36, 1766-1775 (1997). 
20. Descriptions of Hamamatsu C5985 chilled CCD camera and Hamamatsu C4742-95 highresolution digital CCD camera are available at http://usa.hamamatsu.com.

21. The ImageJ software is available at http://rsb.info.nih.gov/ij/

22. The Mathematica software is available at http://www.wolfram.com

23. A.Resnick, "Differential interference contrast microscopy as a polarimetric instrument", Applied Optics, 41, 38-45 (2002).

24. R. Oldenbourg, S. Inoue, R. Tiberio, A. Stemmer, G. Mei, and M.Skvarla, "Standard test targets for high resolution light microscopy”. In: Nanofabrication and Biosystems, H.C. Hoch, L.W. Jelinski, and H.G. Craighead, eds., (Cambridge University Press, New York, 1996), pp.123-138. 


\section{Figure captions:}

Fig.1: Conventional DIC microscope setup: Sc, monochromatic light source; $\mathrm{P}\left(45^{\circ}\right)$, polarizer at $45^{\circ}$ azimuth; $\mathrm{R}\left(\Gamma, 0^{\circ}\right)$, retarder at $0^{\circ}$ azimuth and phase shift $\Gamma$; WP1, Wollaston prism 1 with splitting angle $\varepsilon_{1} ; \mathrm{C}$, condenser lens with focal distance $f_{\mathrm{c}}$; Sp, object under investigation with optical path difference $(\boldsymbol{O P D}=\varphi)$ between points A and B; O, objective lens with focal distance $f_{\text {ob }}$ WP2, Wollaston prism 2 with splitting angle $\varepsilon_{2} ; \mathrm{A}\left(-45^{\circ}\right)$, analyzer at $-45^{\circ}$ azimuth; $\mathrm{E}_{\mathrm{x}}$ and $\mathrm{E}_{\mathrm{y}}$ are polarization components; $\delta$ is shear amount.

Fig.2. Regular DIC image of $4 \mu \mathrm{m}$ diameter glass rods immersed in Permount. DIC shear direction is parallel to AA' (left). Linear scans of normalized intensity in sections A-A' and B-B' are shown on the right.

Fig.3. Computed gradient magnitude image of the glass rods (left). Linear plots of normalized gradient magnitude in sections A-A' and B-B' are shown on the right. The experimental data are indicated by dots. The solid lines show the theoretical results calculated using simplified assumptions (see test).

Fig.4. Computed gradient image of the same group of glass rods with azimuth in pseudocolor. The color wheel in the lower left corner depicts gradient azimuth.

Fig.5. Computed phase of the glass rods (left). Linear scans of normalized phase in sections AA' and B-B' are shown on the right. The experimental data are indicated by dots. The solid lines show the theoretical results.

Fig.6. Regular and computed orientation-independent DIC images of Siemens star and line scans along section AA'. The phase image of the Siemens star was calculated by D.Biggs (AutoQuant Imaging Inc., Troy, NY). 
Fig.7. Regular and computed orientation-independent DIC images of Bovine pulmonary artery endothelial cell. The phase image of the Bovine cell was calculated by D.Biggs (AutoQuant Imaging Inc., Troy, NY).

Fig. 8. DIC images of Bovine pulmonary artery endothelial cell with various shear directions computed from orientation-independent DIC data. 


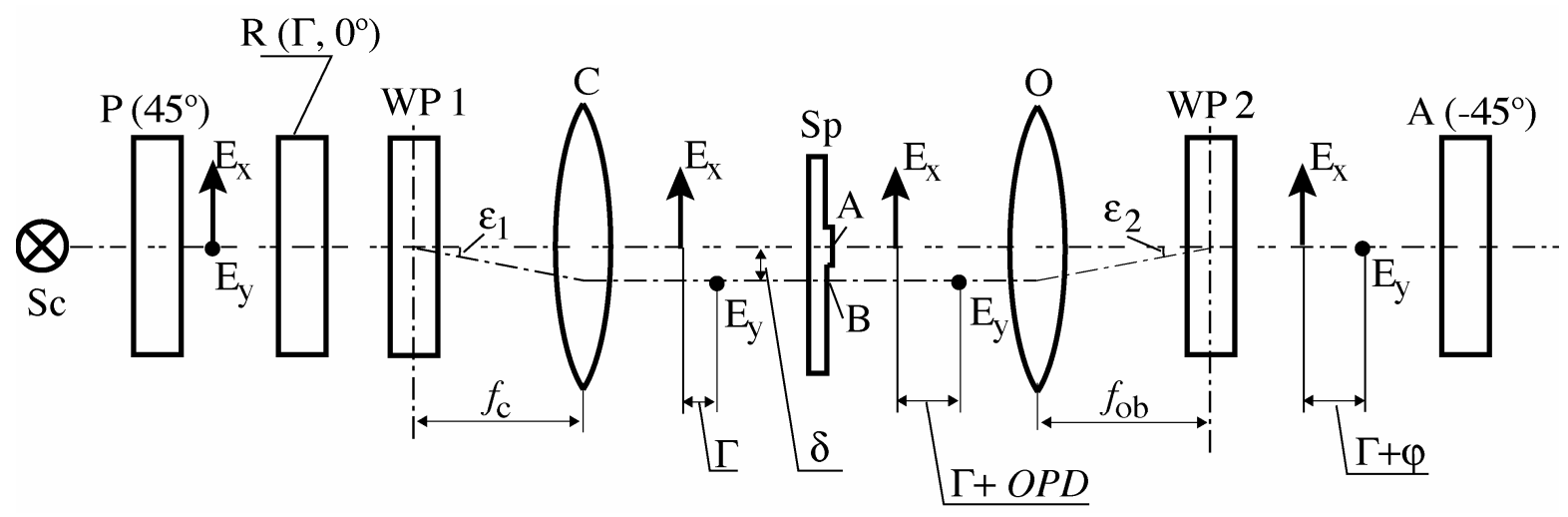

Fig.1
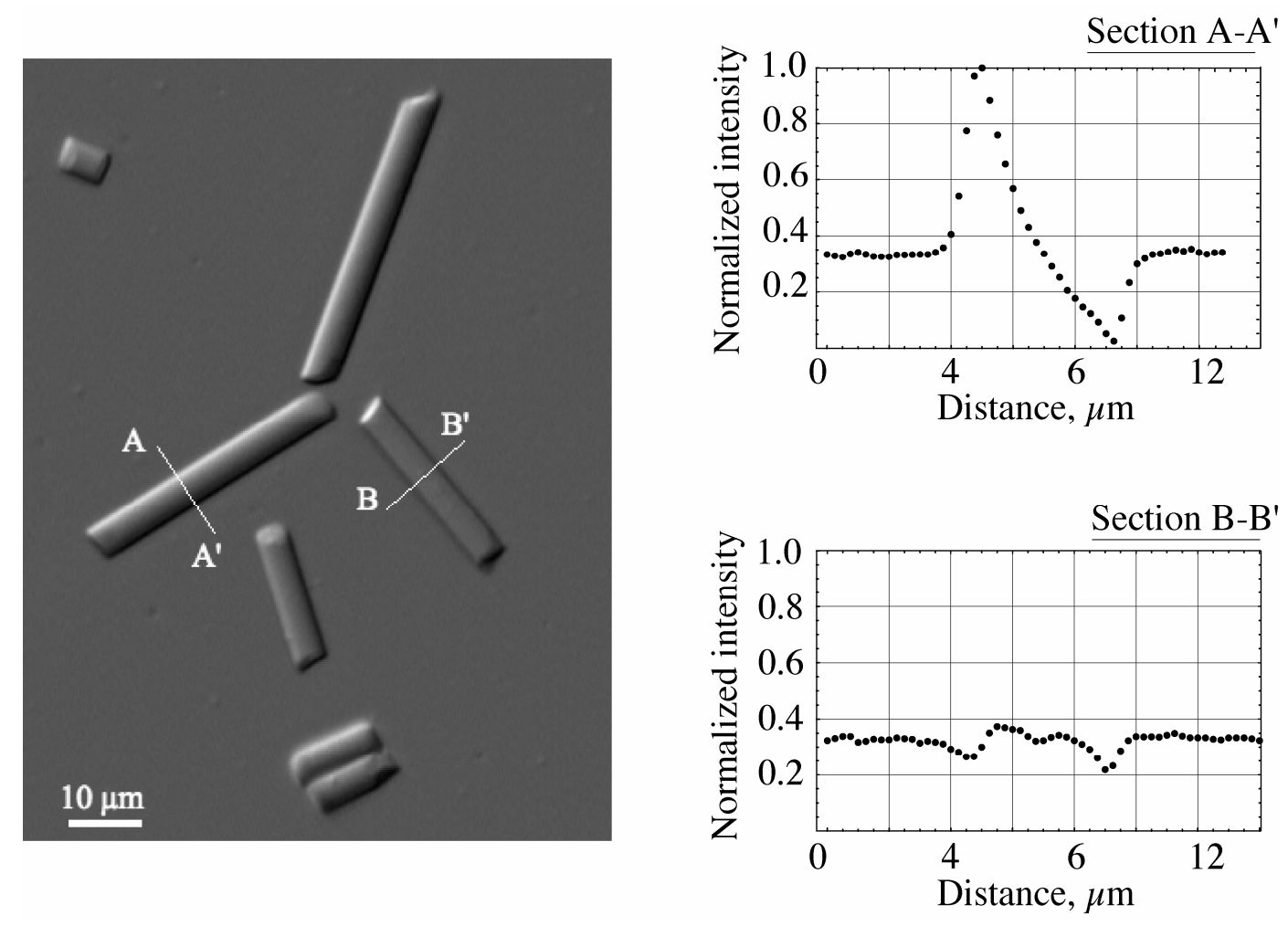

Fig.2 

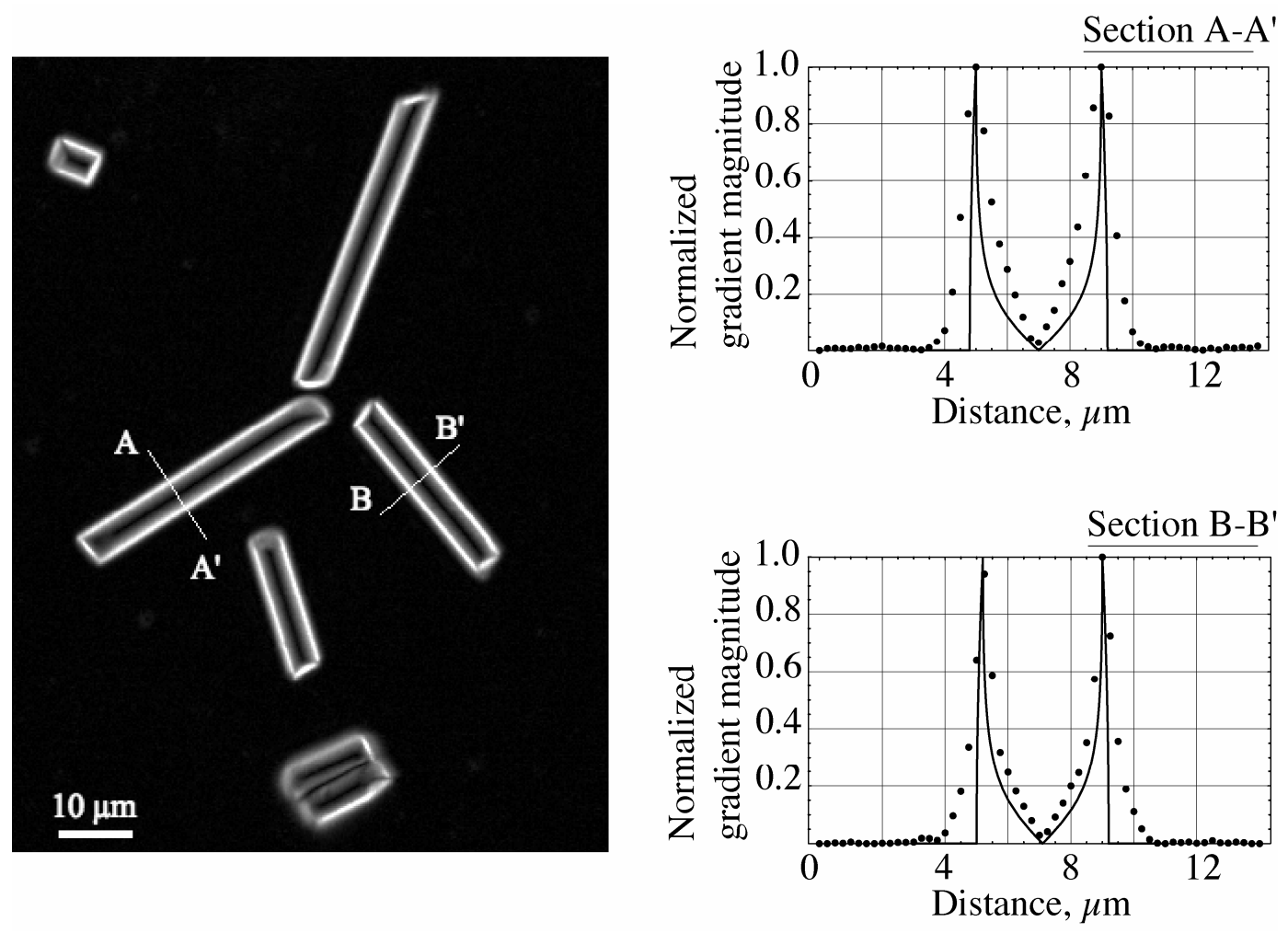

Fig. 3

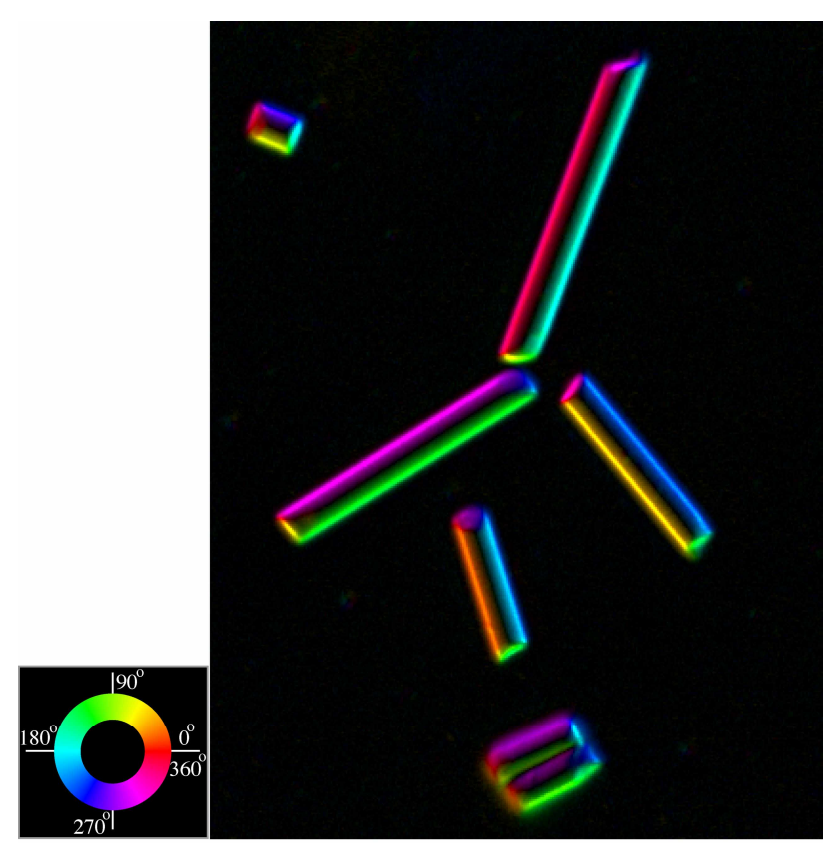

Fig.4 

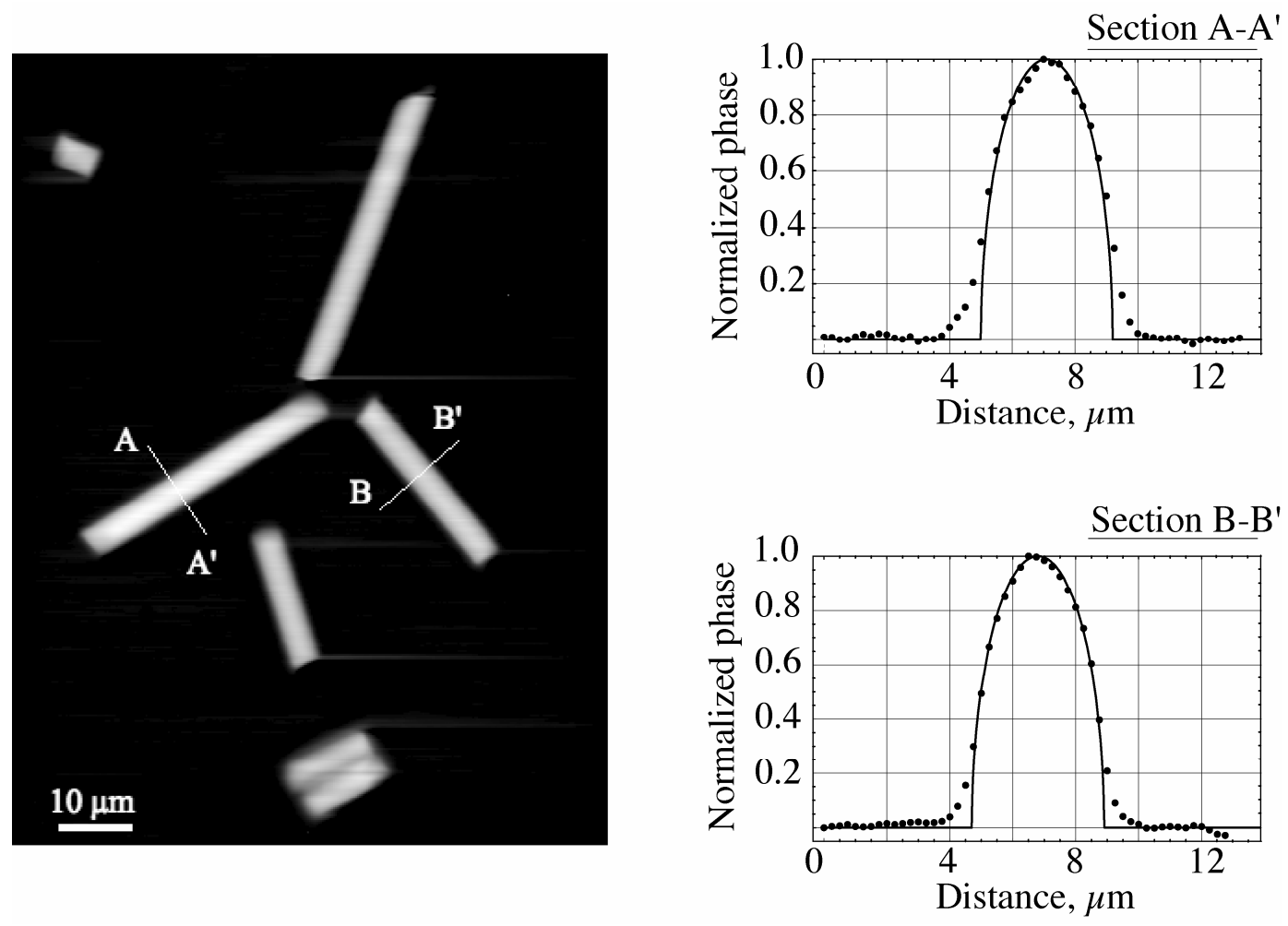

Fig.5

Regular DIC image

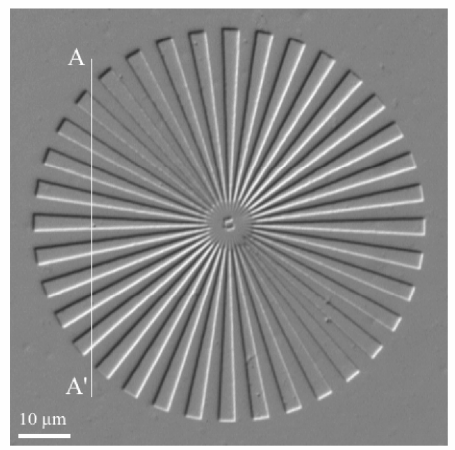

Section A-A

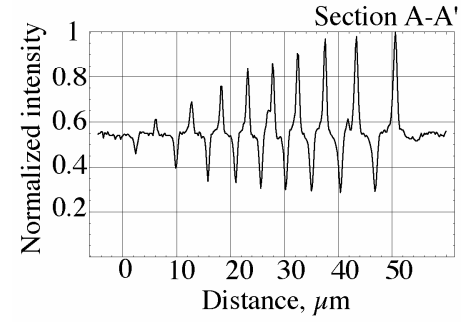

Computed gradient magnitude
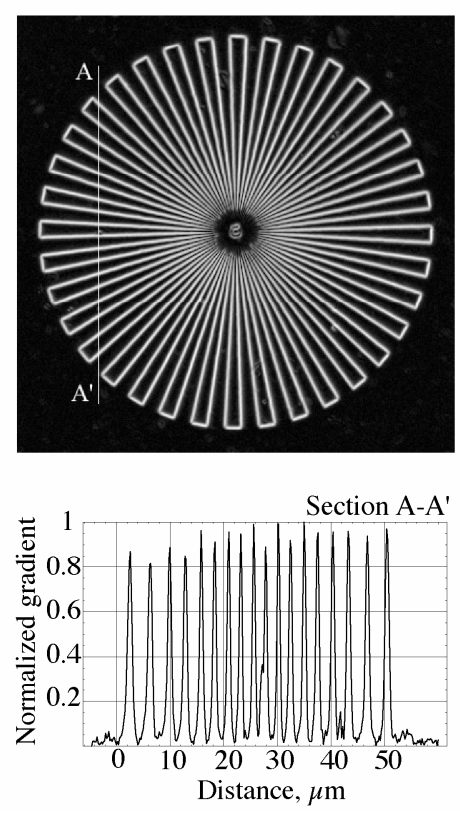

Phase image computed by deconvolution
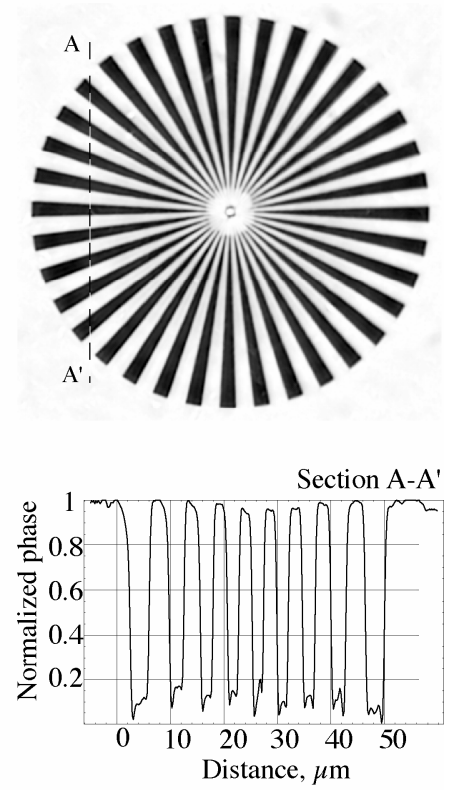

Fig.6 

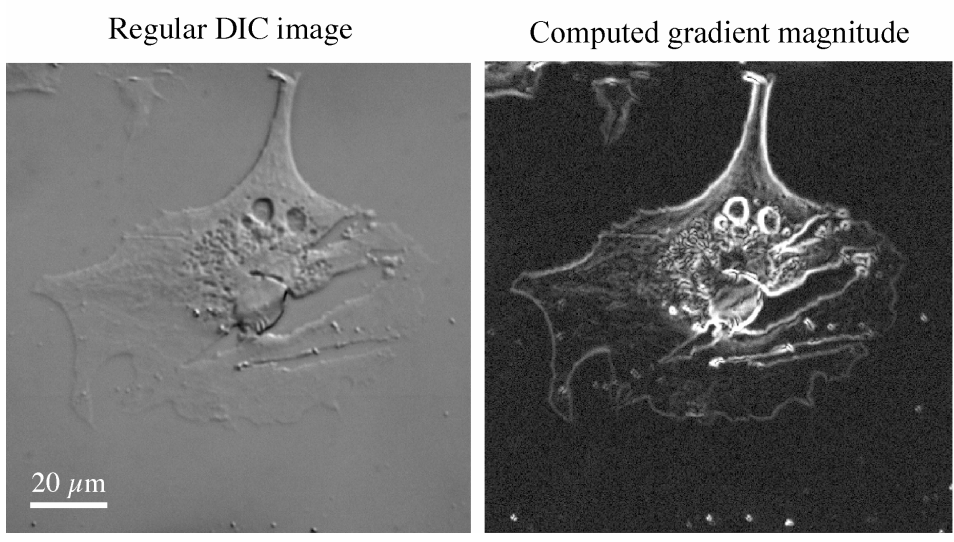

Phase image calculated by deconvolution

Fig.7

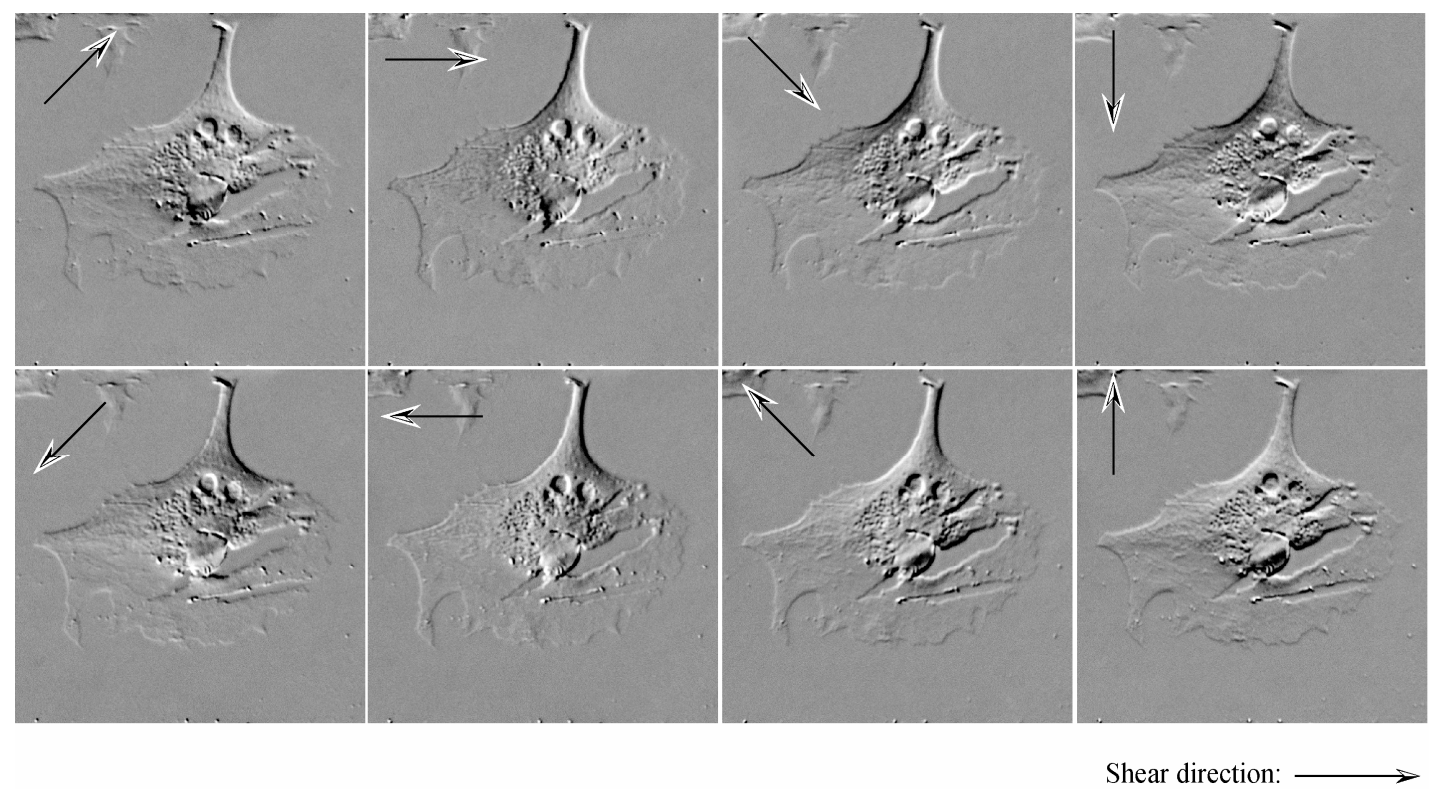

Fig. 8 